\title{
The molecular mechanism of acute liver injury and inflammatory response induced by Concanavalin $\mathrm{A}$
}

\author{
Xiaoxiao Liu' ${ }^{1,2+}$, Ting Yu ${ }^{1,3+}$, Yuzhu Hu ${ }^{1,4+}$, Longzhen Zhang ${ }^{2}$, Junnian Zheng ${ }^{2}$ and Xiawei Wei ${ }^{1^{*}}$ (D)
}

\begin{abstract}
Acute liver injury is a common but urgent clinical condition, and its underlying mechanism remains to be further elucidated. Concanavalin A (ConA)-induced liver injury was investigated in the study. Different from the caspasedependent cell apoptosis in lipopolysaccharide/D-aminogalactose (LPS/D-GaIN) induced liver injury, ConA-induced hepatocyte death was independent on caspase. Increased hepatocytic expressions of mixed lineage kinase domain like (MLKL) and receptor-interacting protein kinase 1 (RIPK1), and higher serum concentration of tumor necrosis factor-a (TNF-a) were noticed in mice with ConA-induced liver injury. Inhibition of RIPK1 protein or deletion of MLKL gene could significantly attenuate the acute liver injury and improve mice survival. Besides, the ConA treatment induced severe hepatic inflammation in wide type (WT) mice in comparison with $\mathrm{Mlkl}^{-1-}$ mice, suggesting the RIPK1-MLKL-mediated hepatocellular necroptosis might participate in the process of liver injury. Moreover, mitochondrial damage associated molecular patterns (DAMPs) were subsequently released after the hepatocyte death, and further activated the p38 mitogen-activated protein kinase (MAPK) pathway, which could be reduced by deletion or inhibition of Toll-like receptor 9 (TLR9). Taken together, our research revealed that ConAinduced acute liver injury was closely related to TNF-a-mediated cell necroptosis, and inhibiting RIPK1 or deleting MLKL gene could alleviate liver injury in mice. The mitochondrial DNA released by dead hepatocytes further activated neutrophils through TLR9, thus resulting in the exacerbation of liver injury.
\end{abstract}

Keywords: Liver damage, Mixed lineage kinase domain like protein, Necroptosis, Mitochondrial DNA, Liver inflammation

\section{Introduction}

As the largest solid organ of human body, liver involves in the synthesis or metabolism of various substances, including carbohydrate, fat, protein, vitamins and some harmful substances. Normal functioning of the liver is essential for maintaining body homeostasis. Liver injury caused by viruses, autoimmune responses, alcohol, drugs or other factors is a serious threat to human health [14]. Clinically, patients diagnosed with acute liver injury

\footnotetext{
* Correspondence: xiaweiwei@scu.edu.cn

${ }^{\dagger}$ Xiaoxiao Liu, Ting Yu and Yuzhu Hu contributed equally to this work.

'Laboratory of Aging Research and Cancer Drug Target, State Key Laboratory of Biotherapy and Cancer Center, National Clinical Research Center for Geriatrics, West China Hospital, Sichuan University, Chengdu, China Full list of author information is available at the end of the article
}

often have poor prognosis and high fatality rate due to the acute onset, severe symptoms and concomitant organ failure $[5,6]$. Although liver transplantation is recognized as the optimal choice for treating acute liver injury, its clinical application is restricted due to the lack of suitable donor liver, high costs and subsequent longterm drug-related side effects $[7,8]$. Hence, it is valuable to investigate the underlying mechanisms of acute liver injury and seek for new therapeutic approach.

As a $\mathrm{T}$ cell polyclonal mitogen, Concanavalin A (ConA) could be accumulated in liver and cause liverspecific acute injury by activating $\mathrm{T}$ cells. Moreover, auto-reactive $\mathrm{T}$ cells and auto-antibodies are commonly observed in autoimmune hepatitis (AIH). The $\mathrm{T}$ cell- 
dependent acute liver injury mice model mediated by ConA was successfully established by Tiges in 1992, which was manifested with acute onset, rapidly increased serum levels of glutamic oxalacetic transaminase (AST) and glutamic-pyruvic transaminase (ALT), liver congestion and large necrosis area under the microscope [9]. Owing to the autoimmune-related feature, ConA induced acute liver injury mice model is considered to be an important experimental model of AIH.

It is believed that acute liver injury is related to the death of hepatocytes and release of inflammatory factors. As a new pattern of cell death, necroptosis is different from necrosis and apoptosis [10]. The cell morphology of necroptosis is similar to that of necrosis, both of which are characterized by cell membranes destruction and cellular swelling, while this necroptosis process is programmed and independent of caspases activation [11, 12]. Studies have shown that the necroptosis relies on the activation of tumor necrosis factor- $\alpha$ (TNF- $\alpha$ ) and other tumor necrosis factor related apoptosis inducing ligand (TRAIL) receptor [13-15]. Furthermore the receptor-interacting proteins (RIP) and mixed lineage kinase domain like (MLKL) protein have been reported to play crucial parts in the course of necroptosis [16, 17]. There are various RIP kinases (RIPK) subtypes, in which the RIPK1 and RIPK3 could regulate the process of necroptosis $[17,18]$. RIPK1 regulates the recruitment of necrosome, which could be blocked by the specific RIPK1 inhibitor necrostatin-1 (Nec-1), thereby inhibiting cellular necroptosis $[10,19]$. RIPK3 has been reported not only to enhance the effect of RIPK1, but also to activate downstream MLKL molecular signals together with RIPK1 [20, 21]. When MLKL protein oligomer translocate to plasma membrane, it could cause the sodium ions inflow, cell membrane rupture and eventually lead to cell death $[10,22]$. Besides, MLKL protein and necrotic complexes are found to be distributed in mitochondria and mitochondria-related membranes, indicating RIPK-MLKL mediated cell necroptosis might be closely associated with mitochondria [23].

The release of endogenous molecules after cell death can activate innate immunity, known as damage associated molecular patterns (DAMPs) [24]. DAMPs regulate the immune system by affecting the function of dendritic cells, macrophages, eosinophils and neutrophils. After trauma, the mitochondrial DAMPs, including mitochondrial DNA (mtDNA) and mitochondrial formyl peptide, are released into circulation and activate the innate immune system. In recent years, the research on mtDNA has attracted much attention [25]. As the genetic substance carried by mitochondria, mtDNA is different from nuclear DNA, but more similar to bacterial DNA, rich in CpG sequences [26, 27]. Studies found that CpG can stimulate the phosphorylation of mitogen activated protein kinase (MAPK) through Toll-like receptor 9 (TLR9), thereby triggering the release of inflammatory factors [28-30]. Moreover, mitochondrial formyl peptides affect neutrophils function by binding to its receptor on the surface of neutrophils [31]. Therefore, these mitochondrial DAMPs are of importance in activating inflammatory response, while the correlation between acute autoimmune liver injury and mitochondrial DAMPs as well as the underlying mechanisms remain largely unknown.

Herein, the ConA-induced acute liver injury mice model was established to explore underlying mechanisms of acute liver injury and inflammatory responses. We infer that the release of mitochondrial DAMPs after TNF- $\alpha$ related hepatocyte necroptosis is critical for causing inflammatory response in acute auto-immune liver injury and the RIPK-MLKL signal pathway might well be a potential therapeutic target in future clinic.

\section{Results}

ConA-induced acute live injury was featured with necrotic liver injury

First, the C57BL/6 mice were intravenously injected with ConA $(20 \mathrm{mg} / \mathrm{kg})$ for establishing the acute liver injury model. Obvious acute liver injury was observed $10 \mathrm{~h}$ after ConA injection (Fig. 1a). The results of Gr-1 immunofluorescence staining showed increased infiltration of inflammatory cells in the liver of ConA-treated mice (Fig. 1b). To clarify the pattern of ConA-induced hepatocyte death, lipopolysaccharide/D-aminogalactose (LPS/D-GalN)-induced liver injury model was established to be a contrast. The hepatocyte death in LPS/D-GalN model are known to be caspase-dependent apoptosis [32]. Intriguingly, the gross pathology in ConA-induced liver injury was significantly different from that in LPS/ D-GalN -induced liver injury (Fig. 1c). The liver from ConA model only showed TUNEL positive, different from both positive staining of TUNEL and cleaved caspase-3 in the liver from LPS/D-GalN model which suggested the ConA-induced liver injury tend to be a caspase-independent necroptosis (Fig. 1d). The cells necroptosis depends on the activation of TNF- $\alpha$, thus TNF- $\alpha$ is thought to be essential for ConA-induced liver injury. To explore the effects of TNF- $\alpha$ in ConAinduced liver injury, the serum level of TNF- $\alpha$ in mice was detected. An obvious increase of TNF- $\alpha$ level was observed in mice serum after ConA injection (Fig. 1e). Moreover, after the intravenous injection of TNF- $\alpha$ protein $(100 \mu \mathrm{g} / \mathrm{kg})$ for $10 \mathrm{~h}, \mathrm{C} 57 \mathrm{BL} / 6$ mice showed significantly more neutrophils infiltration in liver than control mice (Supplementary Fig. 1). Therefore, different from caspase-dependent hepatocyte apoptosis in LPS/D-GalN induced liver injury, ConA-induced hepatocyte death 


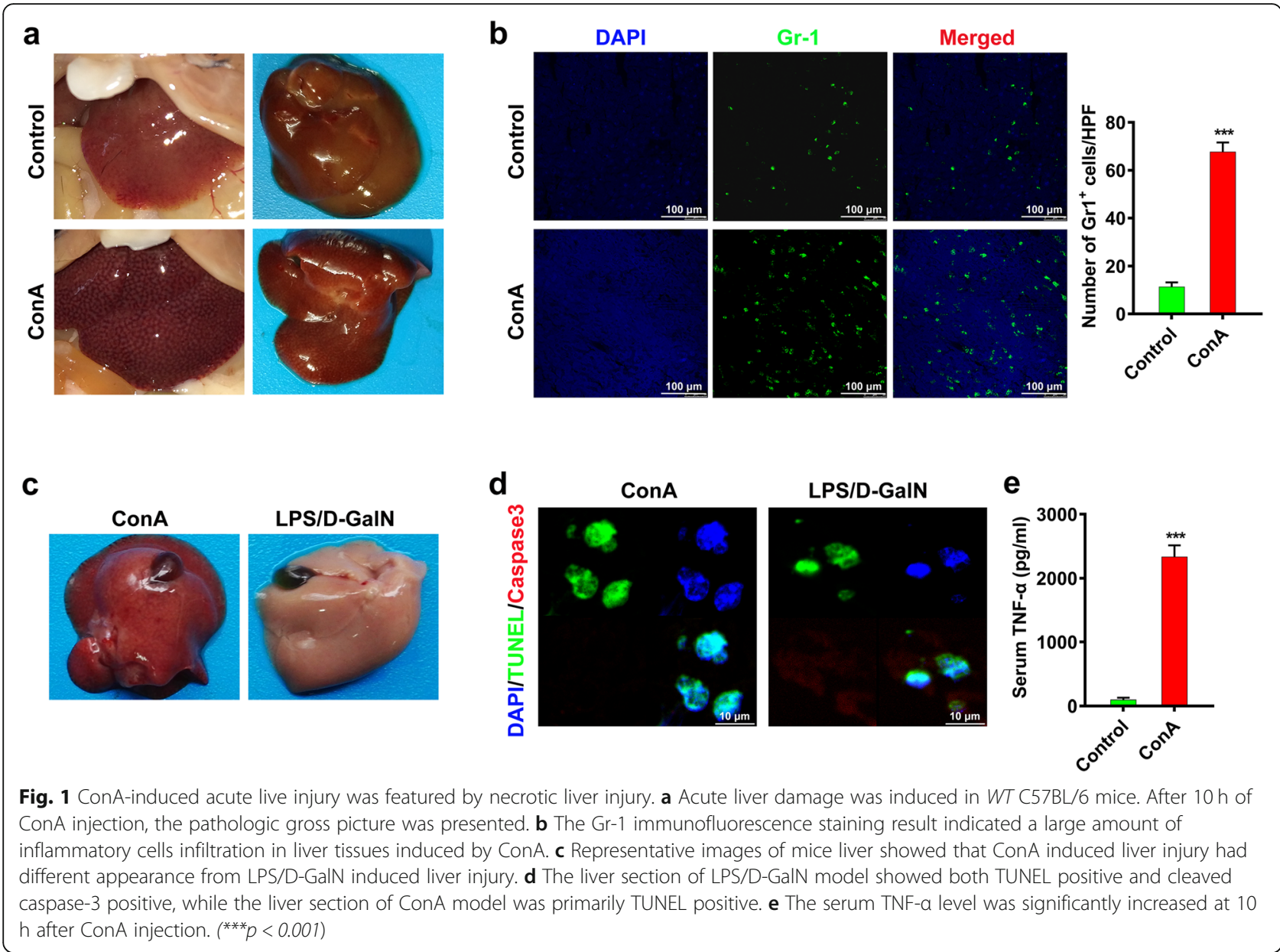

was independent on caspase, suggesting it might be a TNF- $\alpha$-mediated necroptosis.

\section{Role of RIPK1 and RIPK3 in ConA-induced acute liver injury}

As a novel class of kinase, RIPK1 is associated with regulating apoptosis and TNF- $\alpha$ induced necroptosis. RIPK, as a homologous kinase of RIPK1, has been regarded as a key regulator in caspase-independent cell death [20]. The intrahepatic mRNA level of RIPK1 was significantly increased after ConA treatment, while no significant change of the RIPK3 expression level was observed (Fig. 2a). To identify whether RIPK1 was involved in hepatocyte necroptosis, the specific RIPK1 inhibitor Necrostatin-1 (Nec-1) were used for determining the ConA cytotoxicity in L929 cells. The L929 cells pretreated with Nec-1 were resistant to the necroptosis induced by hTNF- $\alpha(\mathrm{T})$, Caspase agonist analogue Smac (S) and caspase-inhibitor QVD-OPH (Q) combination (Fig. 2b). Encouraged by the protective effect of Nec-1 in vitro, we further investigated the effect of RIPK1 inhibition in ConA-induced liver injury model. Half an hour before ConA injection, the mice were intravenously administrated with $\mathrm{Nec}-1(400 \mu \mathrm{g} / \mathrm{kg})$, and the livers were collected after $10 \mathrm{~h}$. The Nec-1 pretreatment successfully alleviated the ConA-induced liver injury and reduced serum levels of ALT, AST and LDH (Fig. 2c, d). What's more, the Nec-1 pretreatment also reduced ConA-induced neutrophilic infiltration in the liver (Fig. 2e). Therefore, the RIPK1 activity but not RIPK3 was relevant to the ConA-induced acute liver injury. We further detected the intrahepatic expression of MLKL, the as yet most known end-stage effector in necroptosis pathway [33]. The injection of ConA significantly increased the intrahepatic expression of MLKL, while pretreatment with Nec-1 showed no significant effect on MLKL expression, indicating MLKL expression was independent of RIPK1 during ConA-induced hepatocyte necroptosis (Fig. 2f).

\section{MLKL may play a vital role in ConA-induced acute liver injury}

Although necroptosis is known to be mediated by RIP kinases and MLKL, the significance of MLKL in acute liver injury remains unclear [33]. To further explore the effect of MLKL on ConA-induced acute liver injury, the 


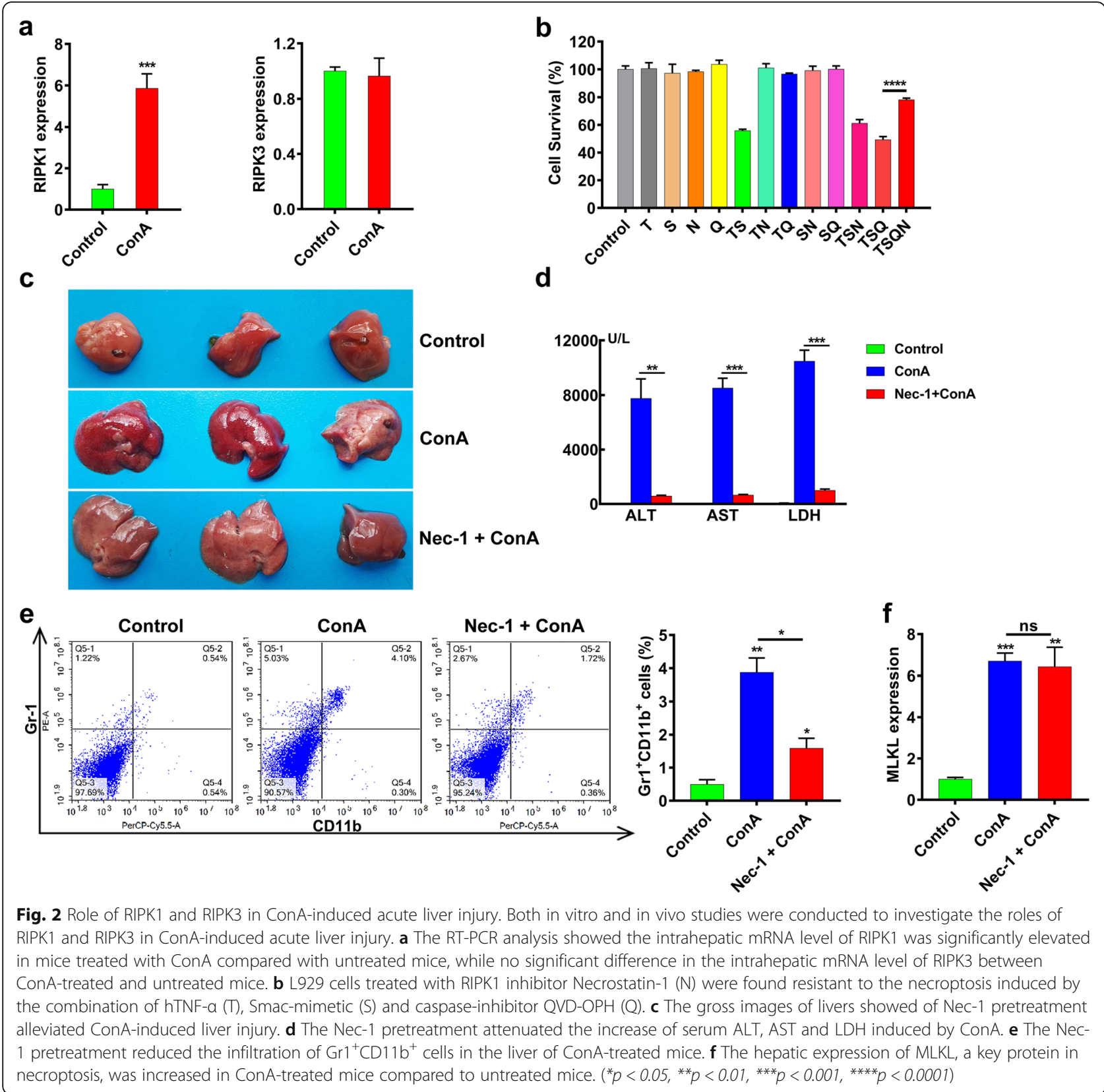

WT mice and $M l k l^{-/-}$mice were intravenously administrated with ConA $(20 \mathrm{mg} / \mathrm{kg})$ and sacrificed $10 \mathrm{~h}$ after injection. Gross and histological results showed siginificantly relieved liver injury in $\mathrm{Mlkl}^{-/-}$mice in comparison with WT mice (Fig. 3a). The ALT, AST and LDH were used to quantify the severity of liver injury. Mice blood samples were collected for serological biochemical analysis at the set time points $(1,3,6$ and 10 h). The results showed the serum levels of ALT, AST and $\mathrm{LDH}$ were increased with time, and the liver injury was much more severe in WT mice when compared to that in $M l k l^{-/-}$mice (Fig. 3b). The WT mice developed severe liver injury (ALT >1000 U/L) at both 6 and $10 \mathrm{~h}$ after ConA injection, while $M l k l^{-/-}$mice suffered mild liver injury. According to TUNEL assay, hepatocyte apoptosis mediated by ConA was obviously reduced in $M l k l^{-/}$mice compared with that in WT mice (Supplementary Fig. 2). Furthermore, the hepatic infiltration of neutrophils was also significantly reduced in $\mathrm{Mlkl}^{-/-}$ mice in comparison with that in WT mice (Fig. 3c), accompanied by significantly lower serum levels of IL-2, IL-6, IL-12, IFN- $\gamma$ and TNF- $\alpha$ (Fig. 3d). Survival analysis 


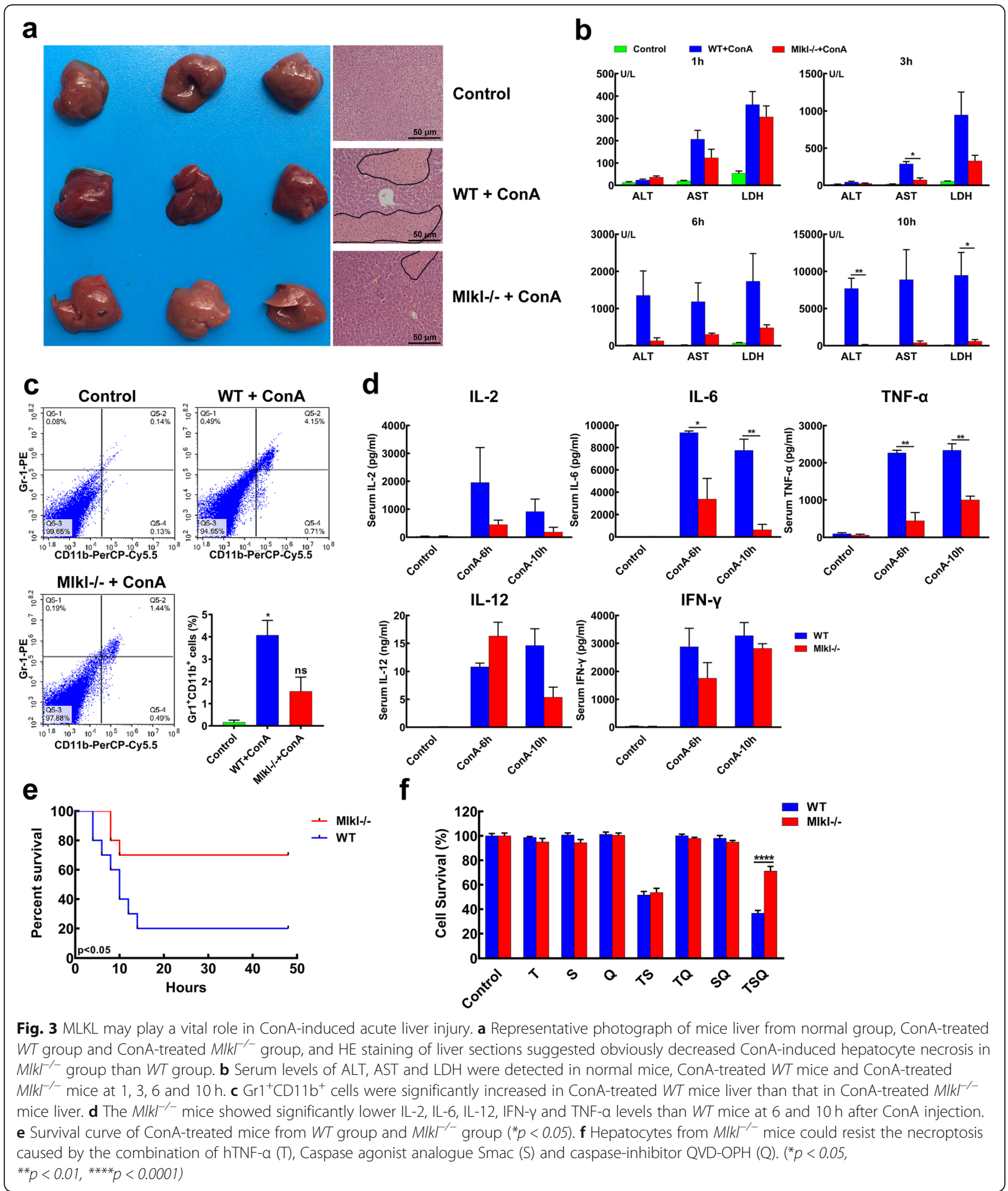

also demonstrated a significantly improved survival of $M l k l^{-/-}$mice in comparison with WT mice after the ConA injection (Fig. 3e). Moreover, primary isolated hepatocytes from $W T$ mice and $M l k l^{-1-}$ mice were used to explore the role of Mlkl in hepatocyte death. As shown in Fig. 3f, hepatocytes from $M l k l^{-/-}$mice were resistant to the necroptosis induced by combination of hTNF- $\alpha$ $(\mathrm{T})$, Caspase agonist analogue Smac (S) and caspase- 
inhibitor QVD-OPH (Q). In short conclusion, these results indicated the deficiency of MLKL gene might play a protective role in ConA-induced acute liver injury.

\section{ConA-induced acute liver injury in mice was mediated by mtDNA release}

The hepatocyte necroptosis induced by ConA was postulated to trigger subsequent liver inflammation. Significantly increased infiltration of esterase-positive neutrophils was observed in the liver of ConA-treated mice (Fig. 4a). The serum levels of elastase and mtDNA were also increased after ConA injection (Fig. 4b). Moreover, the serum level of mtDNA was obviously lower in $\mathrm{Mlkl}^{-/-}$mice in comparison with that in WT mice after ConA treatment (Supplementary Fig. 3a). The ConA treatment also significantly promoted the elastase release in WT mice, while no obvious increase in elastase release was observed in $M l k l^{-/}$mice (Supplementary Fig. 3b). The release of endogenous DAMPs has been speculated to be one possible explanation for the necrotic cells caused inflammatory response. Intracellular components including mitochondria could be served as danger signal to alert the innate immune system [34]. Thereafter, we supposed the mitochondrial leakage from necrotic cells might play a crucial part in ConA-induced acute liver injury. Ten hours after being intravenously injected with the synthetic peptide N-formyl-Met-LeuPhe (fMLF) or extracted mtDNA, the mice were sacrificed. The result showed fMLF, mtDNA, and D+F (mtDNA + fMLF) all induced neutrophil to aggregate in the liver (Fig. 4c). Myeloperoxidase (MPO), a maker of antimicrobial activity, is most abundantly expressed in neutrophil, and the elastase secreted by neutrophils also has important immune functions. We observed that mtDNA could induce the release of MPO and elastase from neutrophils, while fMLF exhibited a mild effect (Fig. 4d). Moreover, the mtDNA stimulated the secretion of matrix metalloproteinase-8 (MMP8) and promoted

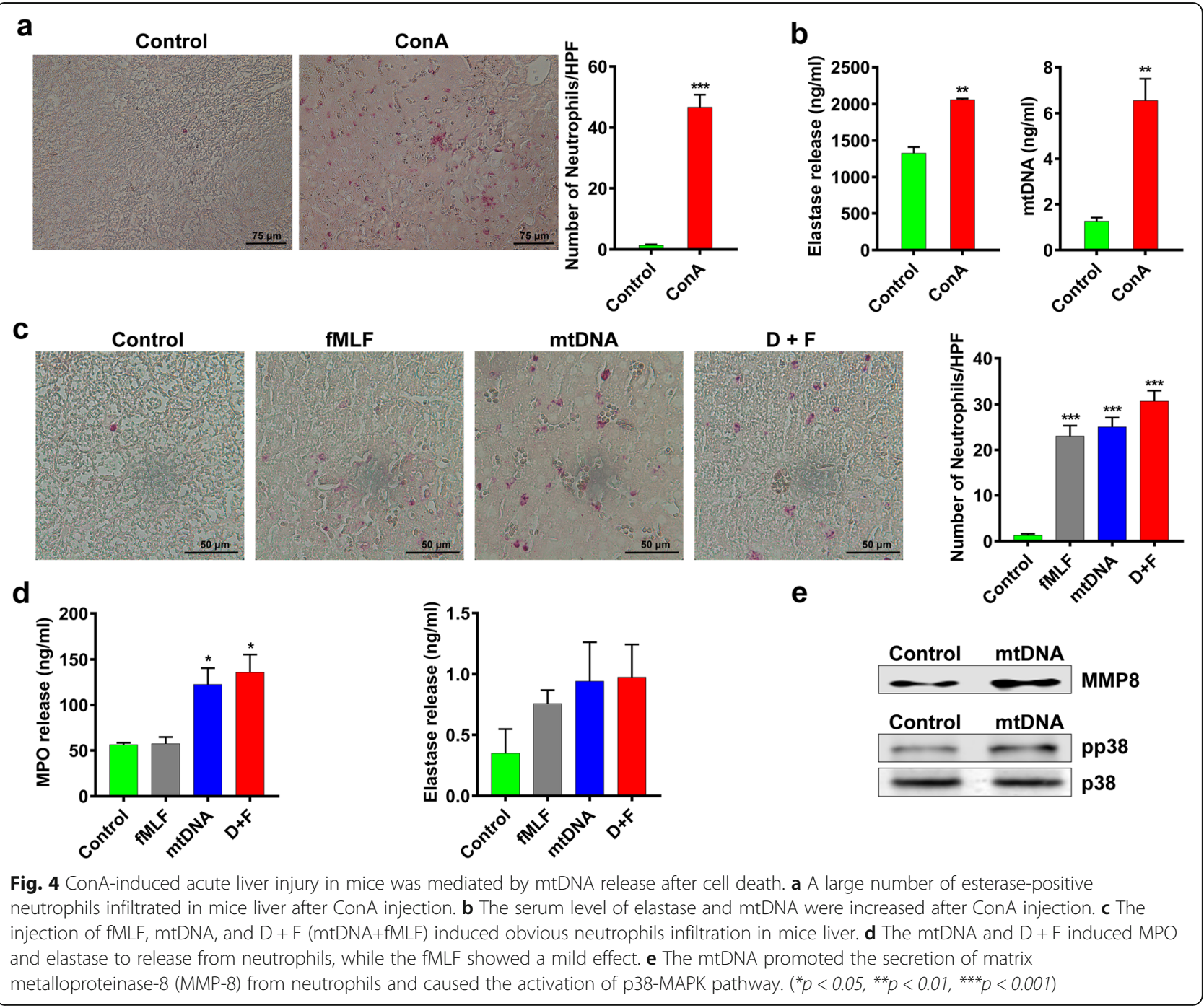


the phosphorylation of p38 protein in neutrophils, suggesting the activation of the $\mathrm{p} 38$ mitogen-activated protein kinase (MAPK) pathway (Fig. 4e). Taken together, the leakage of mitochondrial components from necrotic cells, especially mtDNA, could activate neutrophils through p38-MAPK pathway, stimulate the release of MPO and elastase, and thus evoking the inflammatory response.

\section{ConA could induce inflammatory responses through TLR9 pathway}

Since the preliminary study found that mtDNA might play a role in ConA-induced hepatitis, we tried to figure out the pathway by which the mtDNA played a role. Phosphorylation of MAPK has been reported to trigger subsequent inflammatory responses through TLR9 pathway [35]. Thus, we discussed the role of TLR9 pathway in ConA-induced hepatic inflammation. Both gross and histological observations showed that the liver injury of $T l r-9^{-1-}$ mice treated with ConA was less than that of WT mice treated with ConA (Fig. 5a, b). The serological biochemical analysis confirmed that liver injury was alleviated in $\mathrm{Tlr}-9^{-/-}$mice, with significantly lower levels of liver damage markers (ALT, AST and LDH) (Supplementary Fig. 4a). The survival rate of ConA-treated Tlr$9^{-/-}$mice was significantly improved as well (Fig. 5c). After intravenous injection of mtDNA into WT mice and $\mathrm{Tlr}^{-/-}$mice, a significant reduction in esterasepositive cells infiltration was observed in $\mathrm{Tlr} \mathrm{9}^{-/-}$liver sections (Fig. 5d). Moreover, the result of pretreatment with ODN2088, a TLR-9 antagonist, was in consistence with the above finding, that is, pretreatment with ODN2088 reduced neutrophil infiltration in WT mice (Fig. 5e). Additionally, the treatment of ODN2088 also inhibited the secretion of MMP8 from neutrophils and mtDNA-induced activation of p38-MAPK pathway (Fig. 5f). Therefore, mtDNA can activate neutrophils through TLR-9 pathway. The STING signal pathway has been known to be distinguished from other DNA sensing pathways like TLR9 pathway [36]. Thus, we further investigated whether STING pathway was involved in ConA-induced liver injury. The results showed that ConA still caused severe liver injury in Sting $^{-1-}$ mice. Serum biochemical markers showed no significant relief of liver injury and the mice survival was not prolonged (Supplementary Fig. 4b, c and d). These results

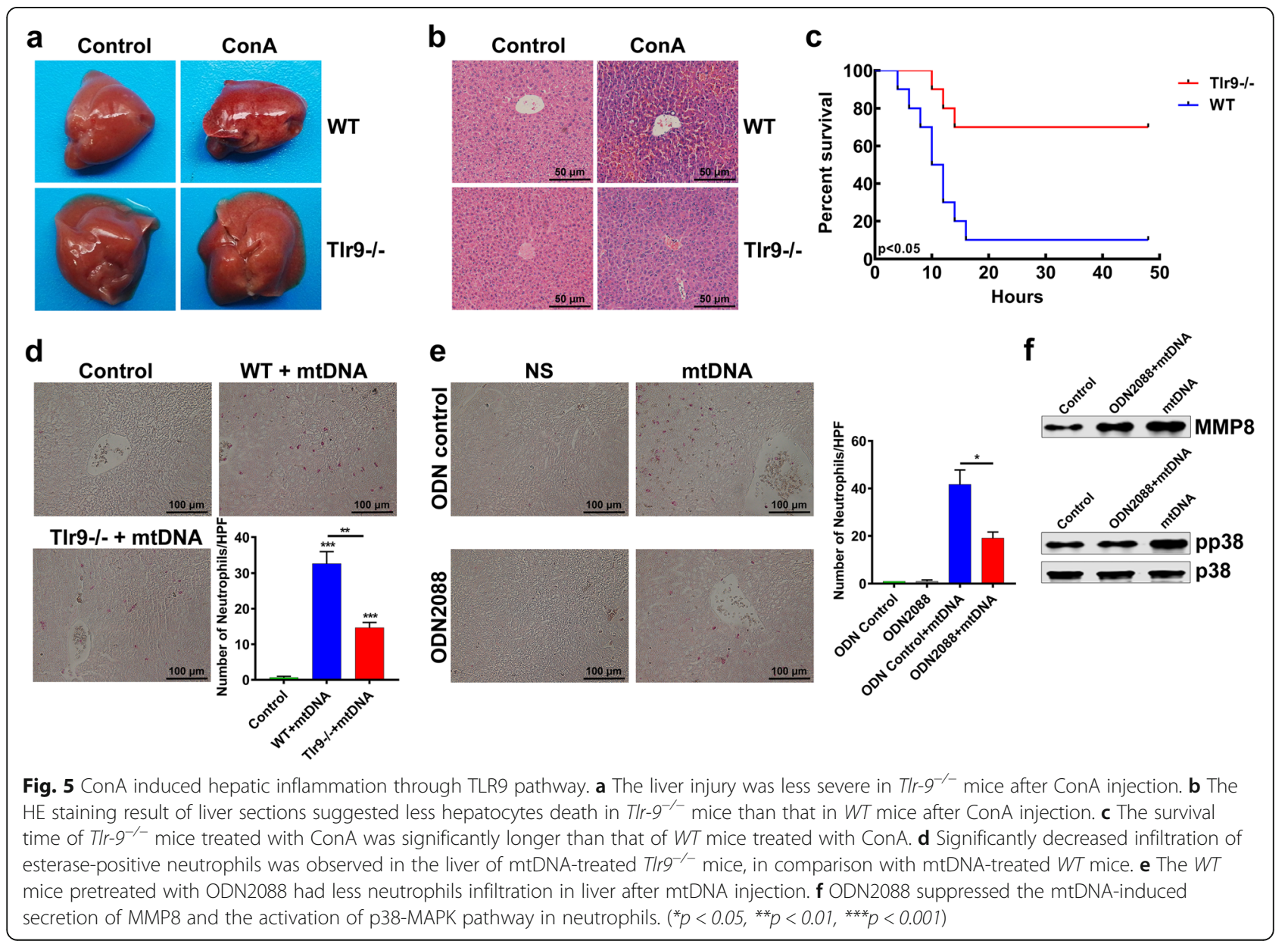


suggested that the subsequent hepatic inflammation caused by ConA might be attributed to the mitochondrial leakage from necrotic hepatocytes, which activate p38-MAPK pathway in neutrophils through TLR-9 pathway.

\section{Discussion}

Acute liver injury is a common but urgent clinical condition, because fulminant liver failure may occur after the exacerbation of acute liver injury and lead to unfavorable prognosis. The underlying molecular mechanisms of acute liver injury remain largely unknown. A deeper understanding of the specific mechanisms will provide strategies to develop more efficient treatment to acute liver injury. The ConA-induced $\mathrm{T}$ cell mediated acute liver injury model is an important experiment model of acute liver injury, which is often applied to investigate the pathogenesis of viral hepatitis and autoimmune hepatitis [37]. Herein, we attempt to investigate and explain the potential mechanisms of ConA-induced acute liver injury.

This study revealed that RIPK1-MLKL is involved in the TNF- $\alpha$ related cell death pathway and plays a critical role in ConA-induced liver injury. The hepatic expression of MLKL was significantly increased in mice treated with ConA, while the deficiency of MLKL gene alleviated the liver injury and improved the survival of mice. Our results found the cell apoptosis in ConA induced liver injury was independent on caspase pathway, which was different from caspase dependent cell apoptosis in LPS/ $\mathrm{D}$-GalN induced liver injury. Moreover, the serum level of TNF- $\alpha$ was substantially increased in ConA-treated mice, which was capable of inducing both cell apoptosis and necroptosis. As an important component in necroptosis, the hepatic expression of RIPK1 was increased in ConA-treated mice and the inhibition of RIPK1 had a protective effect on liver injury. Moreover, RIPK1 inhibition or MLKL deficiency reduced cell death caused by the combination of hTNF- $\alpha$, caspase-activator analogue Smac and caspase-inhibitor QVD-OPH in vitro, suggesting RIPK1 and MLKL participated in TNF- $\alpha$ related cell death in acute liver injury. Furthermore, the mtDNA was found to release extracellular, and resulted in neutrophils activation and pro-inflammatory factors secretion. The ablation of TLR9 not only attenuated hepatic inflammation and alleviated ConA induced liver injury, but also improved the survival of mice.

Plenty of evidence has confirmed the involvement of TNF- $\alpha$ pathway in liver injury process $[38,39]$. Herein, we reported the TNF- $\alpha$-related necroptosis which was mediated by RIPK1 and MLKL contributed to ConA induced acute liver injury. TNF- $\alpha$ has been disclosed to show dichotomous role in cell death process, triggering caspase-8 dependent apoptosis or RIPK1 dependent necroptosis [40]. The cell death would switch to necroptosis rather than apoptosis when deubiquitinated RIPK1 presented with caspase unactivated [41]. Our results showed that cells in liver sections were TUNEL positive but cleaved caspase- 3 negative, indicating this kind of cell death was more likely to be necroptosis. One previous study has reported that the liver injury induced by ConA could not be attenuated by caspase inhibition, which supported our hypothesis. The blockage of necroptosis has been demonstrated to ameliorate tissue damage in atherosclerosis, pancreatitis and systemic inflammatory response $[42,43]$. What's more, study has found RIPK3 or MLKL deficiency partially alleviated liver inflammation in TNF- $\alpha$-dependent multi-organ inflammation that caused by Sharpin-deficiency [44]. In this study, increased hepatic expressions of MLKL and RIPK1 were observed and MLKL deficiency or RIPK1 inhibition exhibited protective roles in acute liver injury process. However, no significant change about the expression level of RIPK3 in liver tissue was observed, which might be owing to intrinsically weak hepatic expression of RIPK3 [45]. All above evidences supported that RIPK1-MLKL mediated cell necroptosis could contribute to ConA induced acute liver injury.

Mitochondrial DAMPs was disclosed to activate innate immunity and cause sterile inflammatory response [46]. Mitochondrial DAMPs in circulation could arouse organ injury mediated by neutrophils [47]. Our previous study has revealed that mitochondrial DAMPs could be released after cell death and triggering pulmonary inflammation through activating neutrophils [48]. Results of this study showed increased hepatic infiltration of neutrophils and increased mtDNA content in mice serum after treating with ConA, thereupon it was inferred that the release of mitochondrial DAMPs after cell necroptosis could partially drive hepatic inflammation. In consistence, further results showed the hepatic mtDNA and fMLF could induce infiltration of neutrophils and improved secretions of MPO, elastase and MMP-8 as well as promoted the phosphorylation of P38. mtDNA recognized by TLR9 was able to trigger inflammatory response and release pro-inflammatory factors including TNF- $\alpha$ or IL- 6 or adhesion molecules [49]. Research found genetic ablation of TLR9 reduced idiopathic liver injury, fibrosis and hepatocellular carcinoma (HCC) in hepatic deletion of TGF- $\beta$-activated kinase 1 (Tak $1 \Delta$ Hep) mice [50]. We revealed the deficiency or inhibition of TLR9 could mitigate hepatic inflammation and restrain the activation of neutrophils, thus reducing the release of inflammatory mediators. TLR9 deficiency successfully abated ConA induced hepatic injury and improved the survival of mice.

In conclusion, we proposed that TNF- $\alpha$-related cell death pathway involved in ConA-induced acute liver 
injury. Hepatocellular necroptosis contributed to the hepatoxicity induced by ConA. Inhibiting RIPK1 protein or deleting MLKL gene could significantly attenuate acute liver injury in mice. What's more, the mitochondrial DAMPs released after cell death could activate neutrophils p38 MAPK pathway through TLR9 pathway, thus promoting pro-inflammatory factors release and resulting in the exacerbation of liver injury. The association between RIPK1 and RIPK3 expression and the relationship of RIPK1-MLKL pathway activation and mtDNA release remain to be elucidated in our future studies. In this contribution, the potential molecular mechanism of acute liver injury and inflammatory response in vivo were elucidated, and our study might provide possible target for clinical treatment of acute liver injury especially autoimmune acute liver injury.

\section{Materials and methods}

\section{Animals}

6-8 weeks old Female C57BL/6 mice were purchased from Beijing Huafukang Biotechnology Co. Ltd. (Beijing, China). The $M l k l^{-/}$mice and $T l r 9^{-/-}$mice were provided by the State Key Laboratory of Biotherapy (SKLB). All animal experiments in this study were approved by the Animal Experimental Ethics Committee of SKLB, Sichuan University (Chengdu, China) and conducted according to the guidelines for the care and use of laboratory animals.

\section{Neutrophils extraction}

Healthy 6-8 weeks old wild type (WT) female C57BL/6 mice were sacrificed and immersed into $75 \%$ ethanol solution for sterilization. The tibia and femur of mice were separated under sterile conditions, and Serum-free RPMI 1640 culture medium was used to flush bone marrow cavity with a $1 \mathrm{ml}$ syringe until bone marrow cavity became white. Gradient separation solution and filtered cells suspension were added into the centrifuge tube in sequence, followed by gradient centrifugation for $0.5 \mathrm{~h}$ at $800 \mathrm{~g}$. Then, the middle layer of cells between two separation solutions was gently pipetted and re-suspended with RPMI 1640 medium containing 10\% FBS for later use. The purity of the extracted neutrophils was about $90 \%$.

\section{Western blot assay}

Cells were collected and lysed with $1 \mathrm{ml}$ Lysis Buffer containing phosphatase inhibitor, protease inhibitor and phenylmethanesulfonyl fluoride (PMSF). The supernatant was quantitatively analyzed and prepared for SDS-PAGE electrophoresis. For analyzing proteins in cell culture supernatant, the supernatant was concentrated by ultrafiltration method. Next, samples were separated by electrophoresis and transferred to PVDF membrane. After blocked by skim milk, the membrane was sequentially incubated with primary antibody (1:1000, Cell Signaling Technology,
USA) and secondary antibody (1:5000, Beyotime, China). Finally, the chemiluminescence reagent was used for protein band visualization.

\section{MTT assay}

L929 cells and primary hepatocytes were separately seeded into 96 -well plates (5000 cells/well) and cultivated overnight. Then, cells were treated with different agents (hTNF- $\alpha$ : $50 \mathrm{ng} / \mathrm{ml}$ (T), Smac-mimetic (S): 500 $\mathrm{nM}$, Necrostatin-1 (N): $50 \mu \mathrm{M}$, QVD-OPH $(\mathrm{Q}): 5 \mu \mathrm{M})$ for $18 \mathrm{~h}$. Then, $20 \mu \mathrm{l}$ MTT solution $(5 \mathrm{mg} / \mathrm{ml})$ was added to each well and incubated for $3 \mathrm{~h}$. The culture supernatant was removed and $150 \mu \mathrm{L}$ DMSO was added to each well. The absorbance was measured at $570 \mathrm{~nm}$ wavelength by a microplate reader.

\section{Flow cytometry analysis}

Liver tissue were cut into slices and suspended in serum-free RPMI1640 culture medium. The tissue suspension was filtered by cell strainer and processed with erythrocyte lysis buffer. The obtained cells were washed with phosphate buffer solution (PBS) and then stained with $\mathrm{Gr} 1$ and $\mathrm{CD} 11 \mathrm{~b}$ fluorescent antibodies for $0.5 \mathrm{~h}$ at $4{ }^{\circ} \mathrm{C}$. Stained cells were re-suspended by PBS. The stained samples were detected by a flow cytometer (Accuri $^{\text {in }}$ C6, BD, USA).

\section{Serum biochemical tests and ELISA assay}

Mice serum was obtained and analyzed for ALT, AST and LDH levels by a biochemical analyzer (Hitachi, Tokyo, Japan). All ELISA procedures were performed referring to the manufacture descriptions (mouse IL2, IL6, IL-12, IFN- $\gamma$ and TNF- $\alpha$ ELISA detection kits from Ebioscience, USA, neutrophil elastase ELISA kits from Cusabio, China and myeloperoxidase (MPO) mouse ELISA kit from Invitrogen, USA). The concentrations of different cytokines in samples were calculated according to their respective standard curves.

\section{Hepatocyte mitochondrial DNA extraction}

Mice were sacrificed and sterilized in 75\% ethanol solution. The liver of mice was isolated and homogenized on ice under sterile condition. Next, the obtained hepatic tissue slurry was processed according to mitochondrial DNA isolation kit (Abcam, Cambridge, UK). The obtained mitochondrial DNA samples was then quantified and stored for further use.

\section{Acute liver injury model establishment}

The mice model of acute liver injury was established by tail vein injection of sterilized PBS that containing 2.5 $\mathrm{mg} / \mathrm{ml} \mathrm{ConA}$ at a dose of $20 \mathrm{mg} / \mathrm{kg}$. 


\section{Immunofluorescence staining of $\mathrm{Gr} 1$}

The frozen liver tissue was cut into slices and fixed with pre-cooled acetone at $4{ }^{\circ} \mathrm{C}$ for 20 mins. The sections were treated with $0.5 \%$ triton-X100 for $30 \mathrm{~min}$ and sealed with goat serum for $0.5 \mathrm{~h}$. Then Gr1 antibody (1:50, BD, USA) was added and incubated at $4{ }^{\circ} \mathrm{C}$ in a wet chamber overnight. Next, the sections were incubated with fluorochrome-conjugated secondary antibody at $37^{\circ} \mathrm{C}$ for $1 \mathrm{~h}$. DAPI was applied to label the cell nuclei. The stained liver sections were observed under a fluorescence microscope.

\section{TUNEL assay}

Liver tissues were prepared as paraffin-embedded sections. The TUNEL staining kit (Promega, WI, USA) was used to detect cell apoptosis. Briefly, the tissue sections were baked at $65^{\circ} \mathrm{C}$ for $2 \mathrm{~h}$ and immersed in xylene, gradient ethanol solution $(100 \%, 95 \%, 85 \%, 70 \%, 50 \%)$ and $0.85 \%$ $\mathrm{NaCl}$ solution. Then, $20 \mu \mathrm{g} / \mathrm{ml}$ protease $\mathrm{K}$ was added and incubated for 8 mins. After fixation with 4\% paraformaldehyde solution, the equilibrium buffer was added to each tissue section for 5-10 $\mathrm{min}$ at room temperature. Next, the reaction mixture containing $\mathrm{TdT}$ was added to incubate for $1 \mathrm{~h}$. At last, all sections were immersed in stop reaction solution for 15 mins and washed by PBS. DAPI was used to label the cell nuclei. The stained liver sections were examined under a fluorescence microscope.

\section{Neutrophil specific esterase staining}

The paraffin embedded liver sections were immersed with xylene and gradient ethanol solution (100\%, 95\%, 85\%, $70 \%, 50 \%)$. The neutrophil specific esterase staining was performed according to manufacture instruction of mouse neutrophil specific esterase staining kit (Sigma, MO, USA). The stained liver sections were examined under a light microscope.

\section{Statistical analysis}

All experimental data analysis was performed by SPSS 17.0 software (SPSS, Inc., Chicago, IL, USA). Data were represented by mean \pm standard error of mean (SEM). Comparison between two groups was analyzed by Student's $t$ test. $P$ value of less than 0.05 was considered to be statistically significant.

\section{Supplementary Information}

The online version contains supplementary material available at https://doi. org/10.1186/s43556-021-00049-w.

Additional file 1: Supplementary Fig. $\mathbf{1 ~ G r} 1^{+} C D 11 b^{+}$cells infiltration in TNF-a treated mice and untreated mice. Supplementary Fig. 2 TUNEL staining of liver sections in $M / \mathrm{kl}^{-/-}$mice and WT mice treated by ConA. Supplementary Fig. $\mathbf{3}$ a The serum level of mtDNA and the elastase release in WT mice and $\mathrm{Mlkl}^{-1-}$ mice treated by ConA. Supplementary Fig. 4 a The serum concentration of ALT, AST and LDH in T/r- $9^{-1-}$ mice and WT mice after ConA injection. b Gross appearance and the HE staining result of liver section in Sting ${ }^{-1-}$ mice and WT mice after ConA injection. c Serum level of ALT, AST and LDH in Sting ${ }^{-1-}$ mice and WT mice after ConA injection. d The survival time of Sting ${ }^{-1-}$ mice and WT mice treated with ConA.

\section{Acknowledgements}

None.

Code availability

Not applicable.

\section{Authors' contributions}

Xiaoxiao Liu, Ting Yu and Yuzhu Hu performed experiment, analyzed data and wrote the paper. Longzhen Zhang and Junnian Zheng edited the manuscript. Xiawei Wei designed the study and revised the manuscript. The authors read and approved the final manuscript.

\section{Funding}

This work was supported by the 2018 Doctoral Project for Innovation and Entrepreneurship of Jiangsu Province and the Natural Science Foundation Youth Project of Jiangsu Province (BK20190989) and the Key R\&D Projects of the Science and Technology Department of Sichuan Province (2020YFS0213).

Availability of data and materials

All data generated or used during this study appear in the submitted article and its supplementary files.

\section{Declarations}

Ethics approval and consent to participate

All animal experiments in this study were approved by the Animal Experimental Ethics Committee of SKLB, Sichuan University (Chengdu, China) and conducted according to the guidelines for the care and use of laboratory animals.

Consent for publication

Yes.

\section{Competing interests}

The authors declared no conflict of interests.

\section{Author details}

${ }^{1}$ Laboratory of Aging Research and Cancer Drug Target, State Key Laboratory of Biotherapy and Cancer Center, National Clinical Research Center for Geriatrics, West China Hospital, Sichuan University, Chengdu, China. ${ }^{2}$ Department of Radiation Oncology, Cancer Center, Affiliated Hospital of Xuzhou Medical University, Jiangsu Center for the Collaboration and Innovation of Cancer Biotherapy, Cancer Institute, Xuzhou Medical University, Xuzhou 221000, China. ${ }^{3}$ Department of Pathology, West China Hospital, Sichuan University, Chengdu, China. ${ }^{4}$ Department of Medical Oncology, Cancer Center, West China Hospital, Sichuan University, Chengdu, China.

Received: 1 February 2021 Accepted: 14 April 2021

Published online: 10 August 2021

References

1. Einar BR, Jayant T, Sombat T, Kamath PS, Naoki T, Schuyler S, et al. Druginduced autoimmune hepatitis: clinical characteristics and prognosis. Hepatology. 2010;138(5):S-807-S. https://doi.org/10.1002/hep.23588.

2. Leise MD, Poterucha JJ, Talwalkar JA. Drug-induced liver injury. Mayo Clin Proc. 2014;89(1):95-106. https://doi.org/10.1016/j.mayocp.2013.09.016.

3. Oketani $\mathrm{M}$, Uto H, Ido A, Tsubouchi H. Management of hepatitis B virusrelated acute liver failure. Clin J Gastroenterol. 2014;7(1):19-26. https://doi. org/10.1007/s12328-013-0447-1

4. Wang S, Pacher P, De Lisle RC, Huang H, Ding WX. A mechanistic review of cell death in alcohol-induced liver injury. Alcohol Clin Exp Res. 2016;40(6): 1215-23. https://doi.org/10.1111/acer.13078. 
5. Khashab M, Tector AJ, Kwo PY. Epidemiology of acute liver failure. Curr Gastroenterol Rep. 2007;9(1):66-73. https://doi.org/10.1007/s11894-0080023-x.

6. Yeoman AD, Westbrook RH, Bernal W, Harrison PM, Heaton ND, Wendon JA, et al. Prognosis of acute severe autoimmune hepatitis (AlH): the role of corticosteroids in modifying outcome. J Hepatol. 2014;61(4):876-82. https:// doi.org/10.1016/j.jhep.2014.05.021.

7. Lee WM, Squires RH, Nyberg SL, Edward D, Hoofnagle JH. Acute liver failure: summary of a workshop. Hepatology. 2010;47(4):1401-15. https://doi.org/1 0.1002/hep.22177.

8. Uribe M, Buckel E, Ferrario M, Godoy J, Blanco A, Hunter B, et al. Epidemiology and results of liver transplantation for acute liver failure in Chile. Transplant Proc. 2003;35(7):2511-2. https://doi.org/10.1016/j.tra nsproceed.2003.09.025

9. Tiegs $\mathrm{G}$, Hentschel J, Wendel A. A T cell-dependent experimental liver injury in mice inducible by concanavalin a. J Clin Invest. 1992;90(1):196-203. https://doi.org/10.1172/JCl115836.

10. Alexei D, Zhihong H, Michael B, Yaqiao L, Prakash J, Noboru M, et al. Chemical inhibitor of nonapoptotic cell death with therapeutic potential for ischemic brain injury. Nat Chem Biol. 2005;1 (2):112-9. https://doi.org/10.103 8/nchembio711.

11. Zhang M, Harashima N, Moritani T, Huang W, Harada M. The roles of ROS and caspases in TRAIL-induced apoptosis and necroptosis in human pancreatic cancer cells. PLoS One. 2015;10(5):e0127386. https://doi.org/10.13 71/journal.pone.0127386.

12. Hannes S, Abhari BA, Fulda S. Smac mimetic triggers necroptosis in pancreatic carcinoma cells when caspase activation is blocked. Cancer Lett. 2016;380(1):31-8. https://doi.org/10.1016/j.canlet.2016.05.036.

13. Francis Ka-Ming C, Joanna S, Bixby JG, Martin F, Lixin Z, Michael A, et al. A role for tumor necrosis factor receptor-2 and receptor-interacting protein in programmed necrosis and antiviral responses. J Biol Chem. 2003;278(51): 51613-21. https://doi.org/10.1074/jbc.M305633200.

14. Liu ZG. Molecular mechanism of TNF signaling and beyond. Cell Res. 2005; 15(1):24-7. https://doi.org/10.1038/s..cr.7290259.

15. Wu W, Liu P, Li J. Necroptosis: an emerging form of programmed cell death. Crit Rev Oncol Hematol. 2012;82(3):249-58. https://doi.org/10.1016/j. critrevonc.2011.08.004.

16. Rohde K, Kleinesudeik L, Roesler S, Löwe O, Heidler J, Schröder K, et al. A Bak-dependent mitochondrial amplification step contributes to Smac mimetic/glucocorticoid-induced necroptosis. Cell Death Differ. 2017;24(1): 83-97. https://doi.org/10.1038/cdd.2016.102.

17. Silke J, Rickard JA, Gerlic M. The diverse role of RIP kinases in necroptosis and inflammation. Nat Immunol. 2015;16(7):689-97. https://doi.org/10.1038/ ni.3206.

18. Jouan-Lanhouet S, Arshad MI, Piquet-Pellorce C, Martin-Chouly C, MoigneMuller GL, Herreweghe FV, et al. TRAIL induces necroptosis involving RIPK1/ RIPK3-dependent PARP-1 activation. Cell Death Differ. 2012;19(12):2003-14. https://doi.org/10.1038/cdd.2012.90

19. Cho Y, Challa S, Moquin D, Genga R, Ray T, Guildford M, et al. Phosphorylation-driven assembly of the RIP1-RIP3 complex regulates programmed necrosis and virus-induced inflammation. Cell. 2009;137(6): 1112-23. https://doi.org/10.1016/j.cell.2009.05.037.

20. Wang H, Sun L, Su L, Rizo J, Liu L, Wang LF, et al. Mixed lineage kinase domain-like protein MLKL causes necrotic membrane disruption upon phosphorylation by RIP3. Mol Cell. 2014;54(1):133-46. https://doi.org/10.101 6/j.molcel.2014.03.003.

21. Orozco S, Yatim N, Werner MR, Tran H, Gunja SY, Tait SWG, et al. RIPK1 both positively and negatively regulates RIPK3 oligomerization and necroptosis. Cell Death Differ. 2014;21(10):1511-21. https://doi.org/10.103 8/cdd.2014.76.

22. Yang $X$, Chao X, Wang ZT, Ding WX. The end of RIPK1-RIPK3-MLKLmediated necroptosis in acetaminophen-induced hepatotoxicity? Hepatology. 2016;64(1):311-2. https://doi.org/10.1002/hep.28263.

23. Lu W, Sun J, Yoon JS, Zhang Y, Zheng L, Murphy E, et al. Mitochondrial protein PGAM5 regulates mitophagic protection against cell necroptosis. PLoS One. 2016;11(1):e0147792. https://doi.org/10.1371/journal.pone.014 7792.

24. Hajime K, Rock KL. How dying cells alert the immune system to danger. Nat Rev Immunol. 2008;8(4):279-89. https://doi.org/10.1038/nri2215.

25. Simmons JD, Lee YL, Pastukh VM, Capley G, Muscat CA, Muscat DC, et al. Potential contribution of mitochondrial Dna damage associated molecular patterns in transfusion products to the development of acute respiratory distress syndrome after multiple transfusions. J Trauma Acute Care Surg. 2017;82(6):1023-9. https://doi.org/10.1097/TA.0000000000001421.

26. Shock LS, Thakkar PV, Peterson EJ, Moran RG, Taylor SM. DNA methyltransferase 1, cytosine methylation, and cytosine hydroxymethylation in mammalian mitochondria. Proc Natl Acad Sci U S A. 2011;108(9):3630-5. https://doi.org/10.1073/pnas.1012311108.

27. Ximiao H, Shu T, Jing J, et al. The most redundant sequences in human CpG island library are derived from mitochondrial genome. Genomics Proteomics Bioinf. 2010;8(2):81-91. https://doi.org/10.1016/S1672-0229(1 0)60009-5.

28. Appledorn D, Patial SA, Godbehere S, Van-Rooijen N, Parameswaran N, Amalfitano A. Adenovirus vector-induced innate inflammatory mediators, MAPK signaling, as well as adaptive immune responses are dependent upon both TLR2 and TLR9 in vivo. J Immunol. 2008;181(3):2134-44. https:// doi.org/10.4049/jimmunol.181.3.2134.

29. Jae-Ho C, Ju-Youn P, Soo-Ki K. Dependence on p38 MAPK signalling in the up-regulation of TLR2, TLR4 and TLR9 gene expression in trichomonas vaginalis-treated HeLa cells. Immunology. 2010;118(2):164-70. https://doi. org/10.1111/j.1365-2567.2006.02347.x.

30. Zhang L, Deng S, Zhao S, Ai Y, Zhang L, Pan P, et al. Intra-peritoneal administration of mitochondrial DNA provokes acute lung injury and systemic inflammation via toll-like receptor 9. Int J Mol Sci. 2016;17(9):1425. https://doi.org/10.3390/ijms17091425.

31. Boudiaf K, Hurtado-Nedelec M, Belambri SA, Marie JC, Derradji Y, Benboubetra $\mathrm{M}$, et al. Thymoquinone strongly inhibits fMLF-induced neutrophil functions and exhibits anti-inflammatory properties in vivo. Biochem Pharmacol. 2016;104:62-73. https://doi.org/10.1016/j.bcp.2016.01. 006.

32. Schattenberg JM, Zimmermann T, Worns M, Sprinzl MF, Kreft A, Kohl T, et al. Ablation of C-FLIP in hepatocytes enhances death-receptor mediated apoptosis and toxic liver injury in vivo. J Hepatol. 2011;55(6):1272-80. https://doi.org/10.1016/j.jhep.2011.03.008.

33. Guenther C, He G-W, Kremer AE, Murphy JM, Petrie EJ, Amann K, et al. The pseudokinase MLKL mediates programmed hepatocellular necrosis independently of RIPK3 during hepatitis. J Clin Invest. 2016;126(11):4346-60. https://doi.org/10.1172/jci87545.

34. West AP, Shadel GS, Ghosh S. Mitochondria in innate immune responses. Nat Rev Immunol. 2011;11(6):389-402. https://doi.org/10.1038/nri2975.

35. Kawasaki T, Kawai T. Toll-like receptor signaling pathways. Front Immunol. 2014;5. https://doi.org/10.3389/fimmu.2014.00461.

36. Barber GN. STING: infection, inflammation and cancer. Nat Rev Immunol. 2015;15(12):760-70. https://doi.org/10.1038/nri3921.

37. Heymann F, Hamesch $\mathrm{K}$, Weiskirchen R, Tacke F. The concanavalin a model of acute hepatitis in mice. Lab Anim-UK. 2015;49(1_suppl):12-20. https://doi. org/10.1177/0023677215572841.

38. Takayuki M, Seiichi C, Hiroshi T, Tohru Y, Hitoshi N, Masataka S, et al. Adiponectin protects LPS-induced liver injury through modulation of TNFalpha in KK-ay obese mice. Hepatology. 2004;40(1):177-84. https://doi.org/1 0.1002/hep.20282.

39. Cheng J, Qinggao D, Neil K. Role of TNF-alpha in ethanol-induced hyperhomocysteinemia and murine alcoholic liver injury. Hepatology. 2004; 40(2):442-51. https://doi.org/10.1002/hep.20309.

40. Vandenabeele P, Galluzzi L, Fau - Vanden Berghe T, Vanden Berghe T, Fau Kroemer G, Kroemer G. Molecular mechanisms of necroptosis: an ordered cellular explosion. Nat Rev Mol Cell Biol. 2010;11(10):700-14. https://doi. org/10.1038/nrm2970.

41. Lixin Z, Nicolas B, David S, Alan C, Jan O, Chan FK, et al. Competitive control of independent programs of tumor necrosis factor receptor-induced cell death by TRADD and RIP1. Mol Cell Biol. 2006;26(9):3505-13. https://doi. org/10.1128/MCB.26.9.3505-3513.2006

42. Lin J, Li H, Yang M, Ren J, Huang Z, Han F, et al. A role of RIP3-mediated macrophage necrosis in atherosclerosis development. Cell Rep. 2013;3(1): 200-10. https://doi.org/10.1016/..celrep.2012.12.012.

43. Zhang $T$, Zhang Y. Cui MA-Ohoo, Jin L, Wang Y, Iv F et al. CaMKII is a RIP3 substrate mediating ischemia- and oxidative stress-induced myocardial necroptosis. Nat Med. 2016;22(2):175-82. https://doi.org/10.1 038/nm.4017.

44. Rickard JA, Holly A, Nima E, Ueli N, Maurice D, Nieves P, et al. TNFR1dependent cell death drives inflammation in Sharpin-deficient mice. Elife. 2014;3. https://doi.org/10.7554/eLife.03464.001 
45. Mark L, Matthias L, Natalie C, Justyna S, Christoph J, Mihael V, et al. RIP3, a kinase promoting necroptotic cell death, mediates adverse remodelling after myocardial infarction. Cardiovasc Res. 2014;103(2):206-16. https://doi. org/10.1093/cvr/cvu146.

46. Chen GY, Gabriel NE. Sterile inflammation: sensing and reacting to damage. Nat Rev Immunol. 2010;10(12):826-37. https://doi.org/10.1038/nri2873.

47. Qin Z, Mustafa R, Yu C, Yuka S, Tolga S, Wolfgang J, et al. Circulating mitochondrial DAMPs cause inflammatory responses to injury. Nature. 2010; 464(7285):104-7. https://doi.org/10.1038/nature08780.

48. Wei $X$, Shao B, He Z, Ye T, Min L, Sang Y, et al. Cationic nanocarriers induce cell necrosis through impairment of $\mathrm{Na}+/ \mathrm{K}+$-ATPase and cause subsequent inflammatory response. Cell Res. 2015;25(2):237-53. https:/doi.org/10.1038/ cr.2015.9.

49. M W. Mitochondrial evolution. Science. 1999;283(5407):1476-1481. doi: https://doi.org/10.1126/science.283.5407.1476.

50. Song IJ, Yang YM, Inokuchi-Shimizu S, Roh YS, Yang L, Seki E. The contribution of toll-like receptor signaling to the development of liver fibrosis and cancer in hepatocyte-specific TAK1-deleted mice. Int I Cancer. 2018;142(1):81-91. https://doi.org/10.1002/ijc.31029.

\section{Publisher's Note}

Springer Nature remains neutral with regard to jurisdictional claims in published maps and institutional affiliations. 